\title{
Comparative Transcriptional Analysis of Lactobacillus plantarum and Its ccpA-Knockout Mutant Under Galactooligosaccharides and Glucose Conditions
}

\author{
Chen Chen, Linlin Wang, Yanqing Lu, Haiyan Yu and Huanxiang Tian* \\ Department of Food Science and Technology, Shanghai Institute of Technology, Shanghai, China
}

OPEN ACCESS

Edited by:

Vittorio Capozzi,

University of Foggia, Italy

Reviewed by:

Margherita Sacco,

University of Campania Luigi Vanvitelli,

Italy

Teresa Zotta,

Italian National Research Council

(CNR), Italy

*Correspondence:

Huanxiang Tian

tianhx@sit.edu.cn

Specialty section: This article was submitted to

Food Microbiology,

a section of the journal

Frontiers in Microbiology

Received: 10 April 2019 Accepted: 25 June 2019

Published: 09 July 2019

Citation:

Chen C, Wang L, Lu Y, Yu H and Tian H (2019) Comparative

Transcriptional Analysis

of Lactobacillus plantarum and lts ccpA-Knockout Mutant Under

Galactooligosaccharides and Glucose Conditions. Front. Microbiol. 10:1584.

doi: 10.3389/fmicb.2019.01584
Galactooligosaccharides (GOS) are documented prebiotic compounds, but knowledge of the metabolic and regulatory mechanisms of GOS utilization by lactic acid bacteria is still limited. Here we used transcriptome and physiological analyses to investigate the differences in the logarithmic growth phase of Lactobacillus plantarum and L. plantarum $\triangle c c p A$ metabolizing GOS or glucose as the sole source of carbohydrate. In total, 489 genes (16\%) were differentially transcribed in the wild-type L. plantarum grown on glucose and GOS and the value is decreased to $7 \%$ due to the loss of $c c p A$. Only $6 \%$ genes were differentially expressed when the wild-type and the $\operatorname{ccpA}$ mutant were compared on GOS. Transcriptome data revealed that the carbon sources significantly affected the expression of several genes, and some of the genes were mediated by CcpA. In particular, lac and gal gene clusters resembled the corresponding clusters in $L$. acidophilus NCFM that are involved in GOS metabolism, indicating that these clusters may be participating in GOS utilization. Moreover, reverse transcription-PCR analysis showed that GOS-related gene clusters were organized in five independent polycistronic units. In addition, many commonalities were found between fructooligosaccharides and GOS metabolism in L. plantarum, including differentially expressed genes involved in oligosaccharide metabolism, conversion of metabolites, and changes in fatty acid biosynthesis. Overall, our findings provide new information on gene transcription and the metabolic mechanism associated with GOS utilization, and confirm that CcpA plays an important role in carbon metabolism regulation in $L$. plantarum.

Keywords: Lactobacillus plantarum, galactooligosaccharides, metabolic regulation, transcriptome, catabolite control protein A

\section{INTRODUCTION}

Prebiotics are non-digestible food ingredients that exert beneficial effect on the host's health by selectively stimulating the growth and activity of health-promoting bacteria in the gastrointestinal tract (GIT) (Andersen et al., 2012; Arnold et al., 2018). Currently, galactooligosaccharides (GOS) are widely accepted as prebiotics (Macfarlane et al., 2008; Monteagudo-Mera et al., 2016). GOS are oligosaccharides with a degree of polymerization between 2 and 10 galactosyl residues and a 
terminal glucose moiety (Iqbal et al., 2010; Van Leeuwen et al., 2016; Azcarate-Peril et al., 2017). Due to the nature of the oligomer and the association of $\beta$-galactoside linkages, GOS act as prebiotic supplements that particularly promote the growth of lactobacilli and bifidobacteria (Andersen et al., 2011; María et al., 2018).

Although it has long been known that oligosaccharides are a prerequisite for the colonization and probiotic activities of lactic acid bacteria (LAB) in the GIT, the understanding of the molecular basis of GOS uptake and metabolism by the Lactobacillus species is quite recent. Using microarray transcriptome analysis, Andersen et al. (2011) identified that the lac gene cluster (LBA1457-LBA1469) participates in GOS utilization. Specifically, GOS is transported intact by a galactoside-pentose-hexuronide (GPH)-type LacS permease and hydrolyzed by two cytoplasmic $\beta$-galactosidases (LacA of the GH42 family and LacLM of the GH2 family) into glucose and galactose. Glucose and galactose are subsequently metabolized via the glycolytic and Leloir pathways, respectively. Interestingly, although the structures of the lac gene cluster differ markedly among LAB (Yong and Klaenhammer, 2015), both lacS and lacA genes exist in LAB, except in L. delbrueckii subsp. bulgaricus, indicating that LacS and $\beta$-galactosidases of the GH42 family coevolved.

In many bacteria, adaptation to changing carbon sources is achieved through a regulatory mechanism called carbon catabolite repression (CCR). This mechanism ensures that the most profitable carbon sources are used first (Akiyama et al., 2015). The catabolite control protein A (CcpA) is a pleiotropic global regulator of CCR in low-GC gram-positive bacteria (Titgemeyer and Hillen, 2002; Fujita, 2009). When bound to its corepressor, Hpr-Ser-P (Presecan-Siedel et al., 1999; Josef et al., 2006), the complex (CcpA-Hpr-Ser46-P) binds to cataboliteresponsive element ( $\mathrm{cre}$ ) sites in the promoter regions of various genes to repress or enhance gene expression (Seidl et al., 2009; Zotta et al., 2012; Kremling et al., 2015). Six cre sites were found in the cluster related to GOS metabolism in L. acidophilus NCFM, suggesting that CcpA is involved in GOS metabolism. However, the exact mechanism is still unknown.

Lactobacillus plantarum is a versatile lactic acid bacterium used for the production of several fermented and functional foods (Enan et al., 1996; Capozzi et al., 2012; Goh and Klaenhammer, 2015; Sankar et al., 2018; Zhao et al., 2019). Several strains, moreover, have been recognized and used as probiotics (Zhang et al., 2009; Ricciardi et al., 2015; Pavli et al., 2016; Yadav et al., 2016; Qian et al., 2017; Papadopoulou et al., 2018; Arena et al., 2019; Wang et al., 2019). CcpA plays a key role in carbon metabolism regulation (Muscariello et al., 2001; Lorquet et al., 2004; Goffin et al., 2006; Siezen et al., 2006), growth performances and stress robustness of L. plantarum (Muscariello et al., 2011; Mazzeo et al., 2012; Zotta et al., 2012). In particular, the metabolic mechanism of fructooligosaccharides (FOS) in L. plantarum has been investigated in our recent studies. These studies showed that CcpA regulates CCR by direct and indirect regulation of FOSrelated clusters in L. plantarum (Chen et al., 2018; Lu et al., 2018). It has also been reported that different L. plantarum strains can effectively utilize GOS (Iqbal et al., 2010; Akiyama et al., 2015); however, the mechanism of GOS metabolism in L. plantarum is not yet clear, and the role of CcpA is still unknown. In this study, the glucose and GOS metabolism of L. plantraum ST-III and its ccpA-knokout mutant (hereafter referred to as Lactobacillus plantarum $\Delta c c p A$ ) was investigated through differential transcriptome and physiological analysis. These results will provide insight into the metabolic pathway and regulation of GOS in L. plantarum and elucidate the role of CcpA in these processes.

\section{MATERIALS AND METHODS}

\section{Organisms, Media, and Growth Conditions}

Lactobacillus plantarum ST-III was isolated from kimchi and obtained from Bright Dairy \& Food Co., Ltd., China. It has many probiotic properties, such as cholesterol removal and strong adhesion to Caco-2 cells (Wang et al., 2011). L. plantarum $\triangle c c p A$ was constructed as described in Chen et al. (2018). Both L. plantarum ST-III and L. plantarum $\Delta c c p A$ were cultivated in de Man-Rogosa and Sharpe (MRS) broth (Merck, Darmstadt, Germany) at $37^{\circ} \mathrm{C}$ in ambient atmosphere under static conditions. For growth experiments, the strains were grown in a chemically defined medium (CDM) (Robert et al., 2000; Teusink et al., 2005) (see Supplementary Table S8) supplemented with filter-sterilized solutions of $1 \%$ (w/v) GOS or glucose. The GOS used in the CDM was provided by Quantum Hi-Tech Biological Co., Ltd. (Guangdong, China) and comprised $\geq 94 \%$ GOS (degree of polymerization, DP 2-8, average DP $\approx 3.5), \leq 7 \%$ lactose, and $\leq 2 \%$ glucose. To exclude the effect of residual sugars on the growth of L. plantarum, a mixture of lactose and glucose equivalent to their content in 1\% GOS was solely added to the CDM as a control. The results showed that these sugars had no effect on the bacterial growth or the subsequent experiments (data not shown).

\section{Fermentation and Sampling}

For growth experiments, overnight cultures of L. plantarum ST-III or L. plantarum $\triangle c c p A$ were transferred with $2 \%(\mathrm{v} / \mathrm{v})$ inoculum into $500 \mathrm{~mL}$ of CDM supplemented with filtersterilized solutions of $1 \%(\mathrm{w} / \mathrm{v})$ GOS or glucose. The mixtures were incubated for $16-18 \mathrm{~h}$ at $37^{\circ} \mathrm{C}$ in a bioreactor (Bioflo model 115, New Brunswick Scientific Co., Edison, NJ, United States) and flushed with sterile air $(0.1 \mathrm{v} / \mathrm{v} \mathrm{min})$, without agitation and controlling the value of $\mathrm{pH}$. During the cells' growth up to the stationary phase, the samples were withdrawn every $2 \mathrm{~h}$ to measure the optical density at $600 \mathrm{~nm}\left(\mathrm{OD}_{600}\right)$ for growth analysis. Maximum specific growth rates $\left(\mu_{\max }\right)$ were calculated through linear regressions of the plots of $\ln \left(\mathrm{OD}_{600}\right)$ versus time during the exponential growth phase (Carvalho et al., 2011). When the $\mathrm{OD}_{600}$ reached 0.65 (early logarithmic phase) and 1.5 (early stationary phase), cultures grown on GOS or glucose were harvested by centrifugation $\left(8,000 \times g, 10 \mathrm{~min}, 4^{\circ} \mathrm{C}\right)$. The cell pellets obtained at $\mathrm{OD}_{600}$ of 0.65 were flash frozen for storage at $-80^{\circ} \mathrm{C}$ for further RNA isolation. The supernatants 
at both sampling points were filtered through a $0.22 \mu \mathrm{m}$ nylon filter (Titan, China). The sugar consumption and production of organic acids were analyzed by high performance anion exchange chromatography (HPAEC) (Chen et al., 2018) and high-performance liquid chromatography (HPLC), respectively, as previously reported ( $\mathrm{Lu}$ et al., 2018). Three replicate fermentations were carried out for each treatment.

\section{RNA Extraction and Transcriptome Analysis}

Total RNA was extracted and treated as previously described (Lu et al., 2018). The quality and quantity of RNA were evaluated using Thermo Scientific Nanodrop 2000 and an Agilent 2100 Bioanalyzer, respectively.

Transcriptome sequencing (RNA-seq) was performed on Illumina X10 (Illumina, Inc., San Diego, CA, United States) as previously described (Chen et al., 2018). Briefly, the total RNA was incubated with DNase I at $37^{\circ} \mathrm{C}$, rRNA was removed, and cDNA libraries were constructed following a PrimeScript RT reagent kit (Takara, Dalian, China).

\section{Reverse Transcription Quantitative Polymerase Chain Reaction (RT-qPCR) Verification}

Eighteen key differentially expressed genes were subjected to RTqPCR to validate the RNA-seq data. The amplifications were performed with designed primers (see Supplementary Table S6) using the 7300 Fast Real-Time PCR System (Applied Biosystems). For RT-qPCR analysis, the generated cDNA was mixed with $0.2 \mathrm{mM}$ gene specific primers (see Supplementary Table S6) in a total volume of $25 \mathrm{~mL}$. The PCR cycling conditions were as follows: $95^{\circ} \mathrm{C}$ for $10 \mathrm{~min}$, followed by 40 cycles of amplification at $95^{\circ} \mathrm{C}$ for $15 \mathrm{~s}$ and $60^{\circ} \mathrm{C}$ for $30 \mathrm{~s}$. All of the samples were measured in triplicate. The relative gene expression data were analyzed by the $2^{-\Delta \Delta \mathrm{Ct}}$ method (Ren et al., 2009) and normalized to the $16 \mathrm{~S}$ rDNA as the reference gene.

\section{Target Gene Structure Analysis}

Total RNA was extracted and reverse transcribed as described above. Primers were designed based on intergenomic regions spanning two clusters of potentially cotranscriptional genes (lac and gal gene clusters) (Supplementary Table S7). The chromosomal DNA and total RNA without reverse transcription were used as the templates for positive and negative controls, respectively.

\section{Bioinformatics Analysis}

Reads generated by each sample were mapped to the genome of L. plantarum ST-III (accession number: CP002222.1) using Bowtie with a default parameter (Langmead et al., 2009). The raw read count for each gene of the two culture samples was analyzed by DEGseq. The MA-plot-based method with the random sampling model in the DEGseq package was then used to calculate the abundance of the expression of each gene in the two culture samples (Wang et al., 2010). In calculations, DEGseq converts the raw read count to reads per kilo bases per million reads (RPKM). The threshold of the $p$-value for this analysis was determined using the false discovery rate (FDR). "FDR $<0.001$ and $\mid$ normalized fold $\mid>1$ " was used as the threshold to judge significance of gene expression differences in the four comparison groups: the wild-type strain grown on GOS versus the wild-type strain grown on glucose, the $\operatorname{ccp} A$ mutant grown on GOS versus the $c c p A$ mutant grown on glucose, the $c c p A$ mutant grown on glucose versus the wild-type strain grown on glucose, the $\operatorname{ccp} A$ mutant grown on GOS versus the wild-type strain grown on GOS.

\section{Statistical Analysis}

Statistical analyses were conducted using SPSS (v. 19.0, IBM SPSS, Chicago, Ill., United States). Duncan's test was used to determine statistical differences. Differences between samples with a $p$-value of $<0.05$ were considered to be statistically significant.

\section{RESULTS}

\section{Growth Profiles of L. plantarum and L. plantarum $\triangle c c p A$ on GOS and Glucose}

Lactobacillus plantarum and L. plantarum $\triangle$ ccpA were cultured in $\mathrm{CDM}$ containing $1 \%$ glucose and $\mathrm{GOS}$ at $37^{\circ} \mathrm{C}$, and their growth profiles are shown in Figure 1. The maximal specific growth rate $\left(\mu_{\max }\right)$ of the GOS-grown wild-type cultures $\left(0.36 \pm 0.02 \mathrm{~h}^{-1}\right)$ was lower than that of glucose-grown wildtype cultures $\left(0.47 \pm 0.01 \mathrm{~h}^{-1}\right)$. Compared with the wild-type

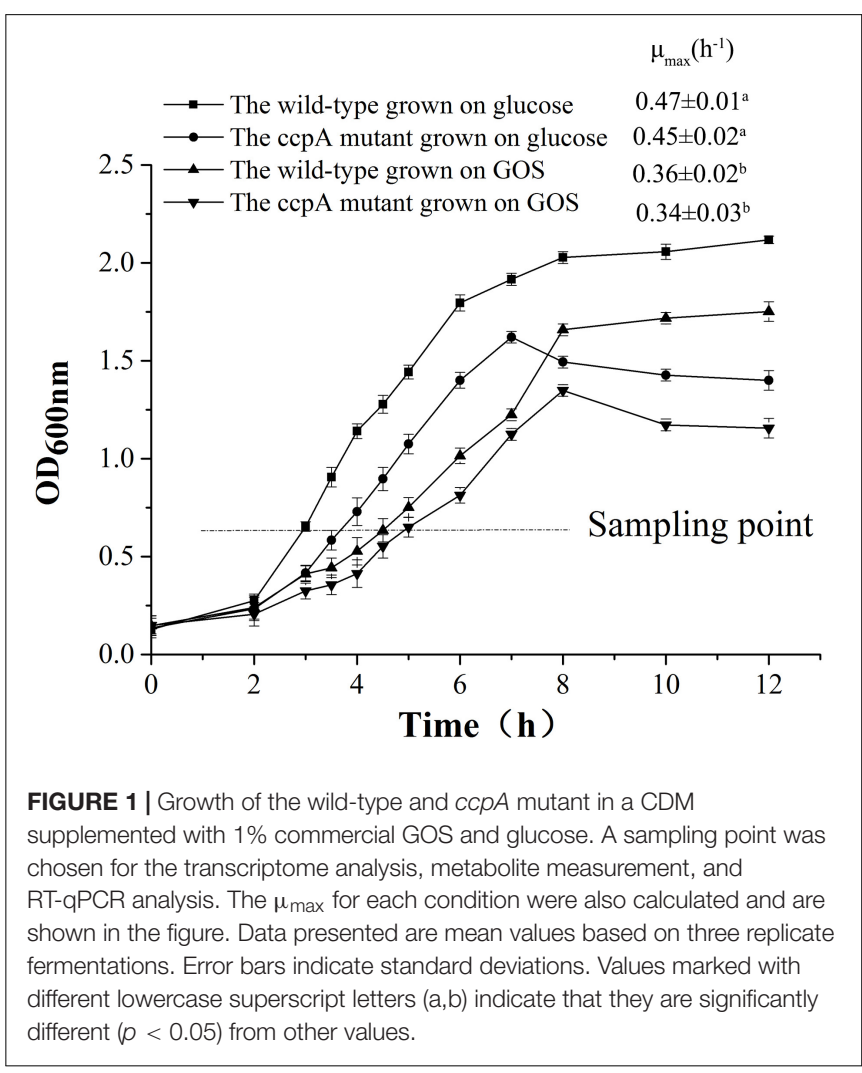




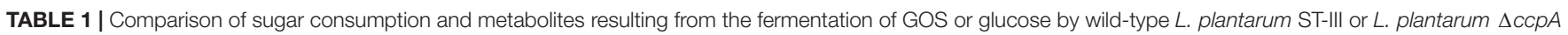
at $\mathrm{OD}_{600}$ of 0.65 and $\mathrm{OD}_{600}$ of $1.5^{1}$.

\begin{tabular}{|c|c|c|c|c|c|c|c|c|}
\hline \multirow[t]{3}{*}{ Conditions } & \multicolumn{4}{|c|}{ Wild-type strain } & \multicolumn{4}{|c|}{ ccpA mutant strain } \\
\hline & \multirow{2}{*}{$\begin{array}{c}\text { Sugar } \\
\text { consumption } \\
\text { (mM) }\end{array}$} & \multicolumn{3}{|c|}{ Metabolite formation (mM) } & \multirow{2}{*}{$\begin{array}{c}\text { Sugar } \\
\text { consumption } \\
\text { (mM) }\end{array}$} & \multicolumn{3}{|c|}{ Metabolite formation (mM) } \\
\hline & & Lactate & Acetate & Formate & & Lactate & Acetate & Formate \\
\hline OD,0.65 Glucose 2,3 & $21.72 \pm 0.51^{\mathrm{Aa}}$ & $15.41 \pm 0.70^{\mathrm{Aa}}$ & $3.03 \pm 0.21^{\mathrm{Aa}}$ & $1.32 \pm 0.10^{\mathrm{Aa}}$ & $20.50 \pm 0.45^{\mathrm{Aa}}$ & $10.63 \pm 0.06^{\mathrm{Ab}}$ & $6.72 \pm 0.12^{\mathrm{Aa}}$ & $1.21 \pm 0.15^{\mathrm{Aa}}$ \\
\hline GOS & $22.43 \pm 0.14^{\mathrm{Aa}}$ & $11.91 \pm 0.22^{\mathrm{Ba}}$ & $6.11 \pm 0.05^{\mathrm{Ba}}$ & $1.20 \pm 0.05^{\mathrm{Aa}}$ & $22.20 \pm 0.29^{A a}$ & $10.31 \pm 0.16^{\mathrm{Aa}}$ & $7.23 \pm 0.12^{\mathrm{Aa}}$ & $1.43 \pm 0.05^{\mathrm{Aa}}$ \\
\hline OD,1.5 Glucose 2,3 & $49.30 \pm 0.23^{A a}$ & $35.32 \pm 1.32^{\mathrm{Aa}}$ & $4.72 \pm 0.05^{\mathrm{Aa}}$ & $1.49 \pm 0.11^{\mathrm{Aa}}$ & $46.08 \pm 0.37^{\mathrm{Aa}}$ & $26.50 \pm 0.78^{A b}$ & $12.41 \pm 0.83^{A b}$ & $1.59 \pm 0.09^{\mathrm{Aa}}$ \\
\hline GOS & $52.65 \pm 0.54^{\mathrm{Ba}}$ & $28.21 \pm 1.12^{\mathrm{Aa}}$ & $11.43 \pm 1.23^{\mathrm{Ba}}$ & $1.61 \pm 0.12^{\mathrm{Aa}}$ & $51.52 \pm 0.46^{\mathrm{Ba}}$ & $26.28 \pm 0.91^{\mathrm{Aa}}$ & $12.92 \pm 0.34^{\mathrm{Aa}}$ & $1.72 \pm 0.21^{\mathrm{Aa}}$ \\
\hline
\end{tabular}

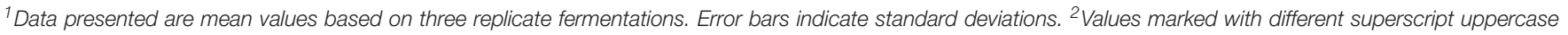
letters (AB) indicate those are statistically significant differences ( $p<0.05$ ) between cells grown on GOS and those grown on glucose at an OD 600 of 0.65 and 1.5 .

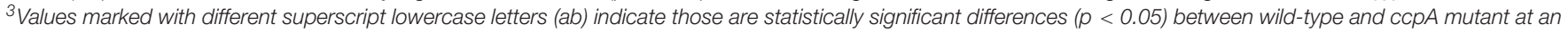
$O D_{600}$ of 0.65 and 1.5 .

strain, L. plantarum $\triangle c c p A$ showed a lower growth rate in glucose $\left(\mu_{\max }, 0.45 \pm 0.02 \mathrm{~h}^{-1}\right)$ and GOS $\left(\mu_{\max }, 0.34 \pm 0.02 \mathrm{~h}^{-1}\right.$ ). Notably, the growth tendency of the two strains grown on the same carbon sources showed subtle differences in the stationary phase. The growth of the wild-type strain increased slightly in glucose and GOS, whereas that of its $c c p A$ mutant showed a slow declining trend.

\section{Fermentation Profiles of $L$. plantarum and L. plantarum $\triangle c c p A$ Grown on GOS and Glucose}

The levels of sugar consumption and metabolite formation during fermentation with L. plantarum ST-III and L. plantarum $\triangle c c p A$ on GOS or glucose are also shown in Table 1. The consumption of GOS is higher than glucose when L. plantarum or L. plantarum $\triangle c c p A$ reached the same value of $\mathrm{OD}_{600}$, and this phenomenon was more significant at an $\mathrm{OD}_{600}$ of 1.5 than at an $\mathrm{OD}_{600}$ of 0.65 . Lactate and acetate are the main end products of fermentation by L. plantarum and L. plantarum $\Delta c c p A$ grown on GOS and glucose. L. plantarum grown on GOS produced less lactate and more acetate than that grown on glucose $(p<0.05)$, which is consistent with the finding of L. plantarum grown on FOS (Lu et al., 2018). In the absence of CcpA, the levels of acetate and lactate did not vary between the strains grown on GOS or glucose. Notably, the $c c p A$ mutant grown on glucose produced more acetate and less lactate than the wild-type strain. This situation also occurred in the presence of GOS, although to a lesser extent. In addition, no difference was observed in the level of formate between the four conditions.

\section{Transcriptome Profiling of Wild-Type and ccpA Mutant Grown on Glucose and GOS}

Based on the growth profiles of the strains on GOS and glucose, the early logarithmic phase was selected for transcriptome analysis. Global transcriptional regulation of wild-type and $\operatorname{ccpA~mutant~in~a~CDM~of~glucose~and~GOS~was~analyzed~}$ by RNA-seq. The statistical data for the transcriptome analysis is summarized in Supplementary Table S1. For L. plantarum ST-III and L. plantarum $\triangle c c p A$ grown on GOS and glucose, the numbers of clean reads obtained were $6.5,7.0,6.6$, and
5.8 million and the mapping rates were $71.50 \%, 73.83 \%$, $69.68 \%$ and $72.19 \%$, respectively. The average read length was 150 bp (see Supplementary Table S2). In addition, the saturation analysis showed that when the number of reads of both samples reached 1 million, the sequencing was saturated (Tan et al., 2015) (Supplementary Figure S1), and the gene coverage indicated sufficient sequencing depth (Lu et al., 2018) (Supplementary Figure S2).

Reads extracted from the RNA-Seq data were mapped into the annotated L. plantarum ST-III genome (NCBI GI: PRJNA493968), and gene expression was quantified as RPKM sequenced. The mean RPKM of the wild-type and ccpA mutant grown on glucose were 419 and 397, respectively, and of wild-type and $\operatorname{ccp} A$ mutant grown on GOS were 417 and 402, respectively (Supplementary Table S1). To investigate the changes in the gene expression of wild-type and $\operatorname{ccp} A$ mutant during fermentation, the standard was set to a fold change of $>2$ and FDR of $<0.001$ (Zheng et al., 2015) (Supplementary Table S1). The overall transcriptome profiles were compared in four pair-wise comparisons to identify genes that were differentially expressed between wild-type and ccpA mutant during GOS or glucose fermentation in CDM. Each differential gene expression profile is described as an XY plot of $\log _{10} \mathrm{RPKM}$ represented by a different color (Supplementary Figure S3). Among the pair-wise comparisons, the transcriptome profiles of the wild-type and $c c p A$ mutant on glucose were compared in our previous study (Lu et al., 2018). In the present study, we focused on the following three pair-wise comparisons to analyze GOS metabolism: between wild-type strain grown on glucose and GOS, between $\operatorname{ccp} A$ mutant grown on glucose and GOS, and between wild-type and $c c p A$ mutant grown on GOS. As per the Kyoto Gene and Genomic Encyclopedia (KEGG) classification, differentially expressed genes were divided into multiple functional categories (Figure 2).

In total, 18 key genes were selected for validating the transcriptome analysis data using RT-qPCR (Supplementary Table S3). Although the magnitude of the genetic variation differed between the results of the two analyses, the RTqPCR results showed similar up- and downregulations to the transcriptome results, confirming the reliability of the transcriptome data. 

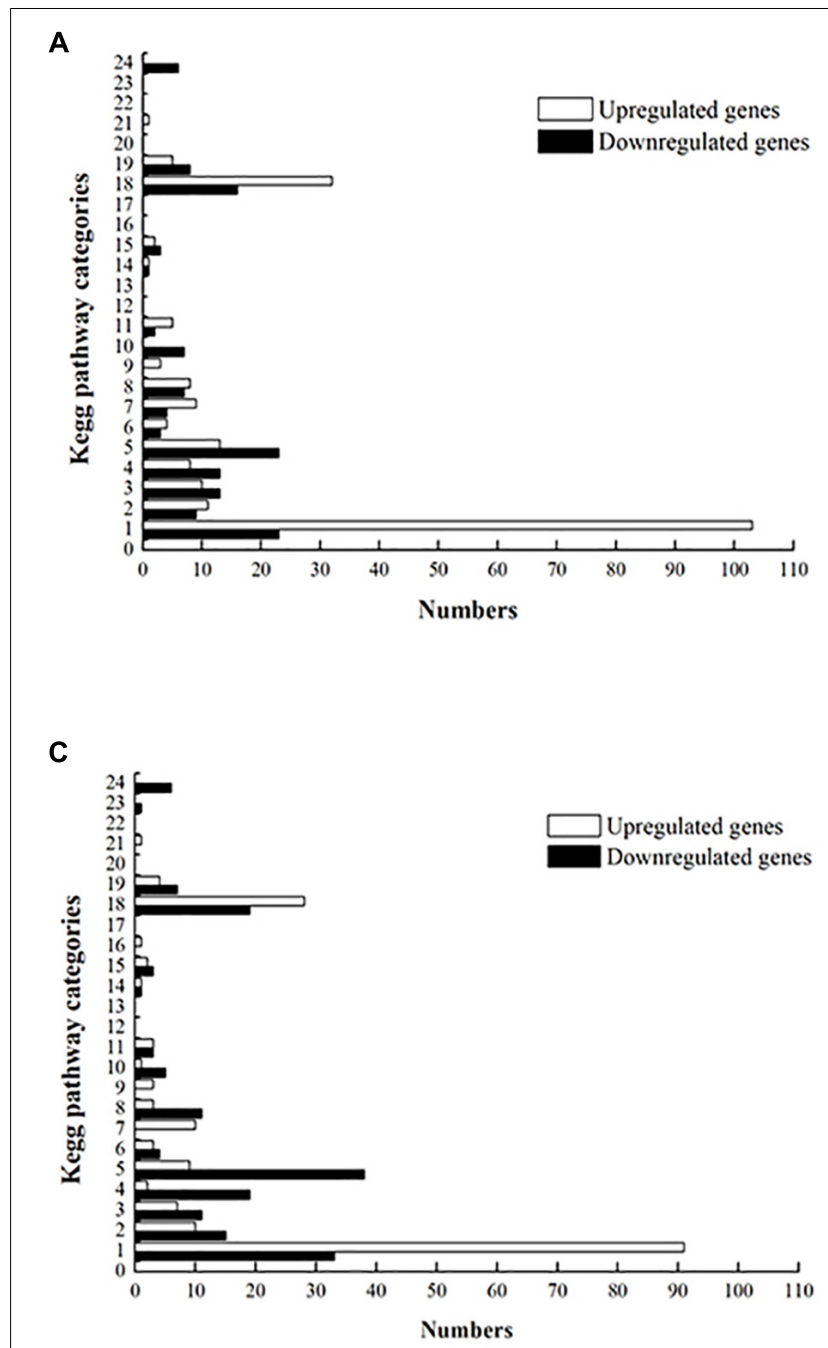

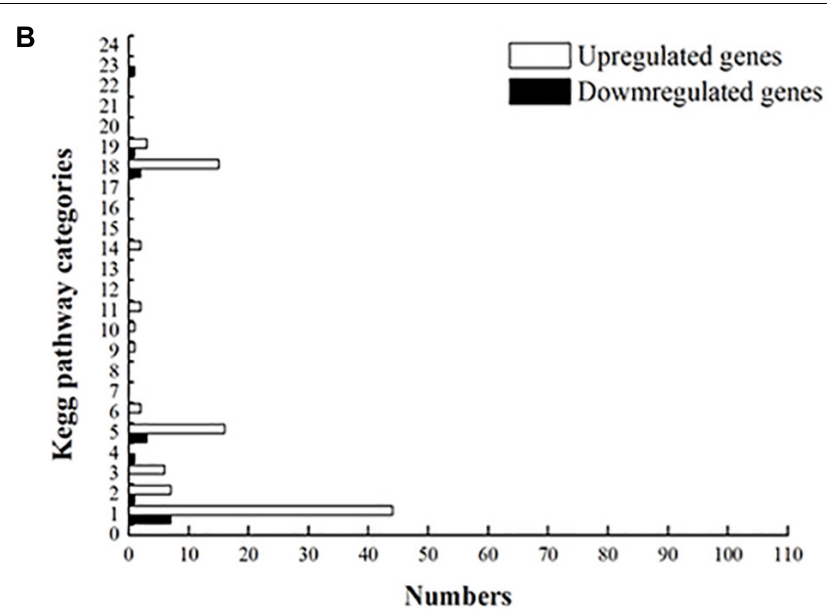

D

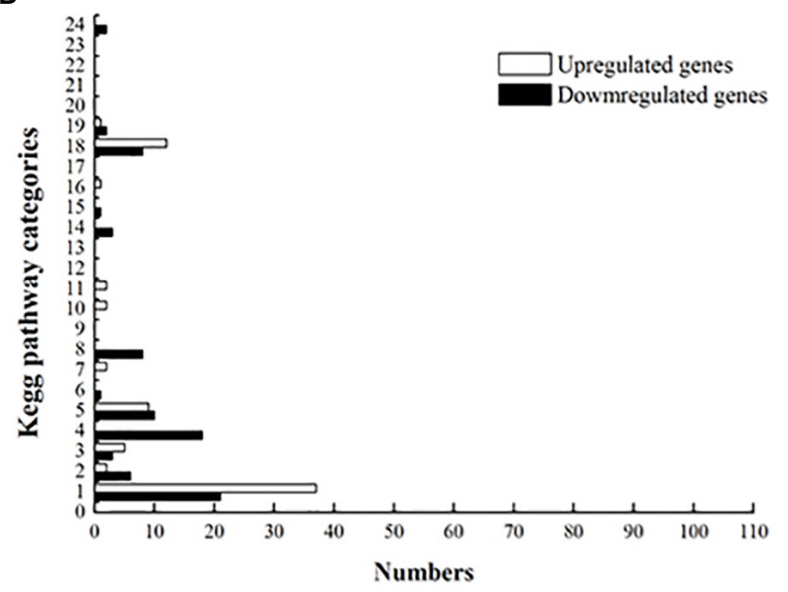

FIGURE 2 | Distribution of upregulated and downregulated genes in the four pair-wise comparisons based on KEGG pathway categories. (A) The ccpA mutant grown on glucose versus the wild-type strain grown on glucose. (B) The ccpA mutant grown on GOS versus the wild-type strain grown on GOS. (C) The wild-type strain grown on GOS versus the wild-type strain grown on glucose. (D) The ccpA mutant grown on GOS versus the ccpA mutant grown on glucose. KEGG pathway categories: 1, carbohydrate metabolism; 2, energy metabolism; 3, lipid metabolism; 4, nucleotide metabolism; 5, amino acid metabolism; 6, metabolism of other amino acids; 7, glycan biosynthesis and metabolism; 8 , metabolism of cofactors and vitamins; 9 , metabolism of terpenoids and polyketides; 10 , biosynthesis of other secondary metabolites; 11, xenobiotic biodegradation and metabolism; 12, enzyme families; 13, transcription; 14, translation; 15, folding, sorting, and degradation; 16, replication and repair; 17, RNA family; 18, membrane transport; 19, signal transduction; 20, signaling molecules and interaction; 21, transport and catabolism; 22, cell motility; 23, cell growth and death; 24, cellular community - prokaryotes.

\section{Comparison Between the Wild-Type Strain Grown on Glucose and GOS}

Supplementary Table S1 lists the genes differentially expressed between wild-type strain grown on glucose and GOS. In total, 489 genes ( $16 \%$ of all genes) were found to be differentially expressed, 254 of which were upregulated and 235 were downregulated (Figure 3A). In the three pair-wise comparisons of this study, this result indicates the largest number of genetic changes. Among the differentially expressed genes, 181 genes were classified into specific expression pathways based on the KEGG database. Notably, most of the differentially expressed genes (35\%) were involved in carbohydrate metabolism, including the phosphotransferase system (PTS), galactose metabolism, pyruvate metabolism, fatty acid biosynthesis, and other such processes (Figure 4).

Notably, 30 PTS genes were differentially expressed in this comparison, reflecting potential changes in the uptake of glucose, galactose, fructose, and cellobiose. In comparison with the wildtype strain grown on glucose, one operon of mannose-specific transporter (LPST_C0484, LPST_C0486) was downregulated (2.03-2.26-fold) and the following six genes were upregulated (2.03-32.67-fold) by GOS: mannitol-specific transporter (LPST_C0187, LPST_C0189), cellobiose-specific transporter (LPST_C0363, LPST_C0366, LPST_C2476-LPST_C2477), ascorbate-specific transporter (LPST_C2890-LPST_C2892), galactosamine-specific transporter (LPST_C2175-LPST_C2177), 
A

$$
\text { GOS / glucose }
$$

206048 upregulted genes wild-type

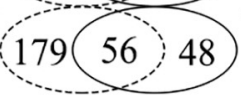

B

$\operatorname{ccp} A$ mutant / wild-type

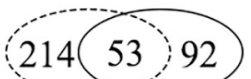

glucose

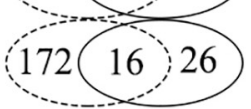

GOS

FIGURE $\mathbf{3}$ | Venn diagrams of the number of genes differentially expressed in the four pair-wise comparisons. (A) Number of genes differentially expressed in response to GOS compared with glucose of the wild-type (dotted line) and cсpA mutant (full line). (B) Number of genes differentially expressed in ccpA mutant compared to the wild-type strains, grown on glucose (dotted line) and GOS (full line).

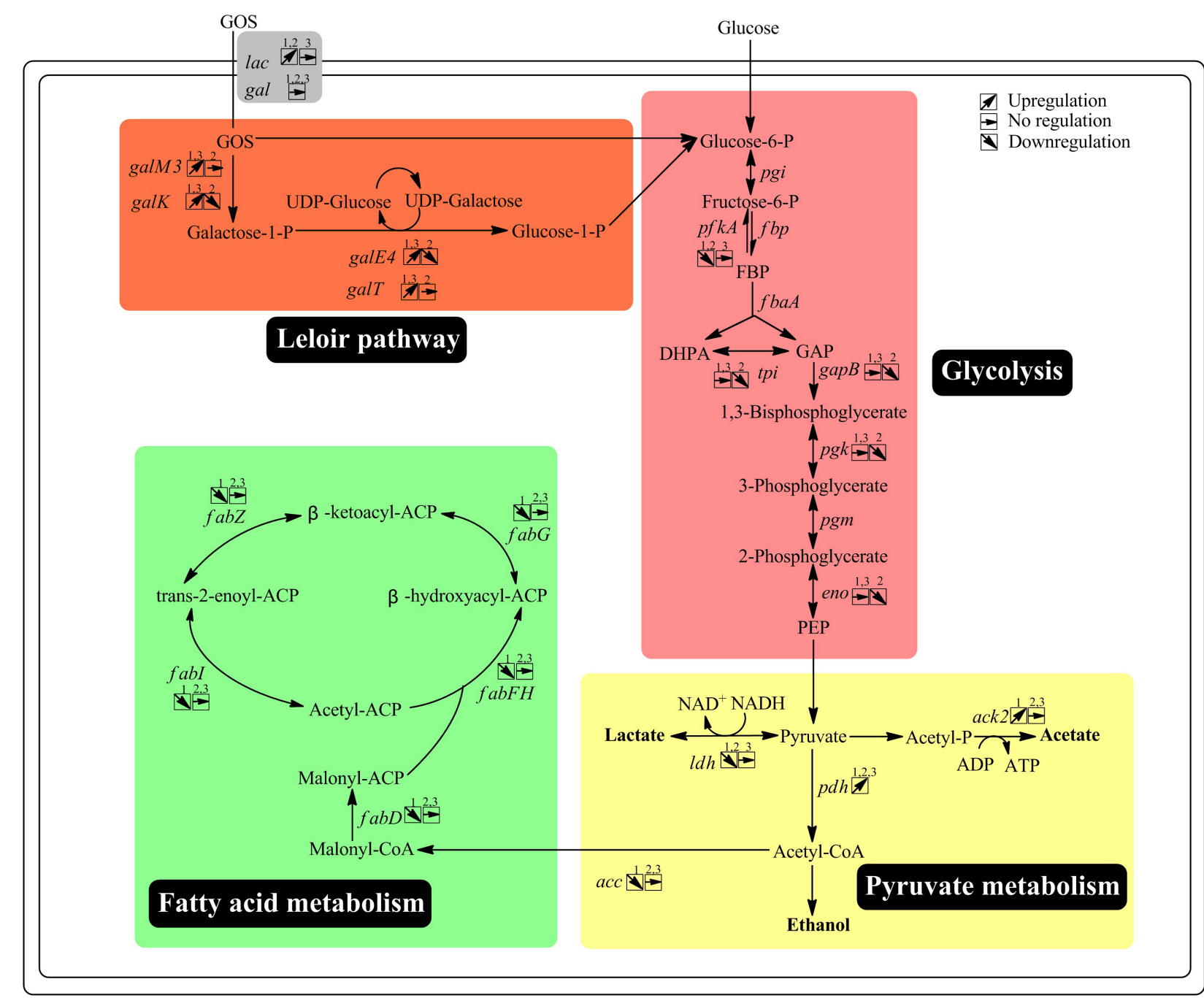

FIGURE 4 | Overview of the key genes with altered expression and their related pathways via transcriptome analysis. 1, The wild-type strain grown on GOS versus the wild-type strain grown on glucose; 2 , the ccpA mutant grown on GOS versus the ccpA mutant grown on glucose; 3 , the ccpA mutant grown on GOS versus the wild-type strain grown on GOS. Gene annotation downloaded from NCBI: galM3, aldose 1-epimerase; galK, galactokinase; galE4, UDP-glucose 4-epimerase; galT, UDPglucose-hexose-1-phosphate uridylyltransferase; pgi, glucose-6-phosphate isomerase; fbp, fructose-1,6-bisphosphatase; pfkA, 6-phosphofructokinase; fbpA, fructose-bisphosphate aldolase; tpi, triose-3-phosphate isomerase; gapB, glyceraldehyde-3-phosphate dehydrogenase; pgk, phosphoglycerate kinase; pgm, phosphoglycerate mutase; eno, enolase; pyk, pyruvate kinase; Idh, L-lactate dehydrogenase; pfl, pyruvate formate-lyase; pdh, pyruvate dehydrogenase; pox, pyruvate oxidase; pta, phosphotransacetylase; ack, acetate kinase; acc, acetyl-CoA carboxylase; fabD, ACP-S-malonyltransferase; fabF, 3-oxoacyl-ACP synthase II; fabH, 3-oxoacyl-ACP synthase III; fabG, 3-oxoacyl-ACP reductase; fabZ, (3R)-hydroxymyristoyl-ACP dehydratase; fabl, enoyl-ACP reductase. 
galactitol-specific transporter (LPST_C2897-LPST_C2898), and glucitol/sorbitol-specific transporter (LPST_C2982LPST_C2983) (Supplementary Table S4). These findings suggest that GOS and its derivatives modify the expression of genes involved in the PTS.

The expression of genes related to pyruvate metabolism and fatty acid metabolism was also altered by GOS metabolism. The genes encoding pyruvate dehydrogenase (LPST_C1775LPST_C1778), pyruvate formate lyase (LPST_C2728) and pyruvate oxidases (LPST_C2161, LPST_C2933) were found to be upregulated in the presence of GOS in this study, while the genes encoding acetyl-CoA carboxylase (LPST_C1533, LPST_C1535LPST_C1537), L-lactate dehydrogenase (LPST_C0295), and pyruvate kinase (LPST_C1523) were significantly downregulated. Differential expression of these genes was associated with pathways in which lactate is converted to acetate and formate, consistent with our previous findings (Chen et al., 2015). Furthermore, in accordance with our previous study, the expression of 10 genes (LPST_C1327-LPST_C1328, LPST_C1330-LPST_C1338) involved in fatty acid biosynthesis showed at least 3.87-fold downregulation in the presence of GOS compared with their expression in the presence of glucose (Supplementary Table S5) (Chen et al., 2015).

As indicated by the transcriptome data, GOS induced significant upregulation (71.51-148.06-fold) in the expression of a cluster (LPST_C2839-LPST_C2841; designated as the lac cluster) comprising genes encoding a GPH permease (LacS1), a $\beta$-galactosidase (LacA), and a transcriptional regulator
(LacR1). In addition, the expression of another related gene cluster(LPST_C2849-LPST_C2858; designated as the gal cluster) increased by 2.93-284.05-fold (Supplementary Table S4). The organization of these two clusters (lac and $g a l$ ) was compared between L. plantarum ST-III and L. acidophilus NCFM by BLASTp analysis to investigate the similarity between their structures (Figure 5). The identified GPH permease LacS1 (LPST-C2839) of L. plantarum ST-III showed 60\% protein sequence identity with the lactose permease of $L$. acidophilus NCFM. Similarly, the two $\beta$-galactosidases LacA (LPST-C2840) and LacLM (LPST-C2853-LPST-C2854) of L. plantarum STIII showed $53 \%$ and $64 \%$ protein sequence identity with their counterparts in L. acidophilus NCFM, respectively. The genes involved in Leloir pathway, including galT (LPST-C2850), galE4 (LPST-C2851) and galK (LPST-C2852), exhibited 55\%, $59 \%$ and $60 \%$ similarity between the two strains, respectively. However, two components, melA (LPST-C2855) and lacS2 (LPST-C2856), which encodes an alpha-galactosidase and a major facilitator superfamily transporter, appeared only in L. plantarum. These results indicate that the clusters in $L$. plantarum and L. acidophilus NCFM have similar structures with a high degree of homology, suggesting that the lac and gal clusters are involved in GOS transport and utilization in L. plantarum.

Furthermore, successive overlapping RT-PCR amplifications of polycistronic operon structures were performed for the analysis of the cotranscription of 13 genes (Figure 6). As previously described, cDNA obtained by reverse transcription of the total RNA extracted from GOS-induced culture was used as

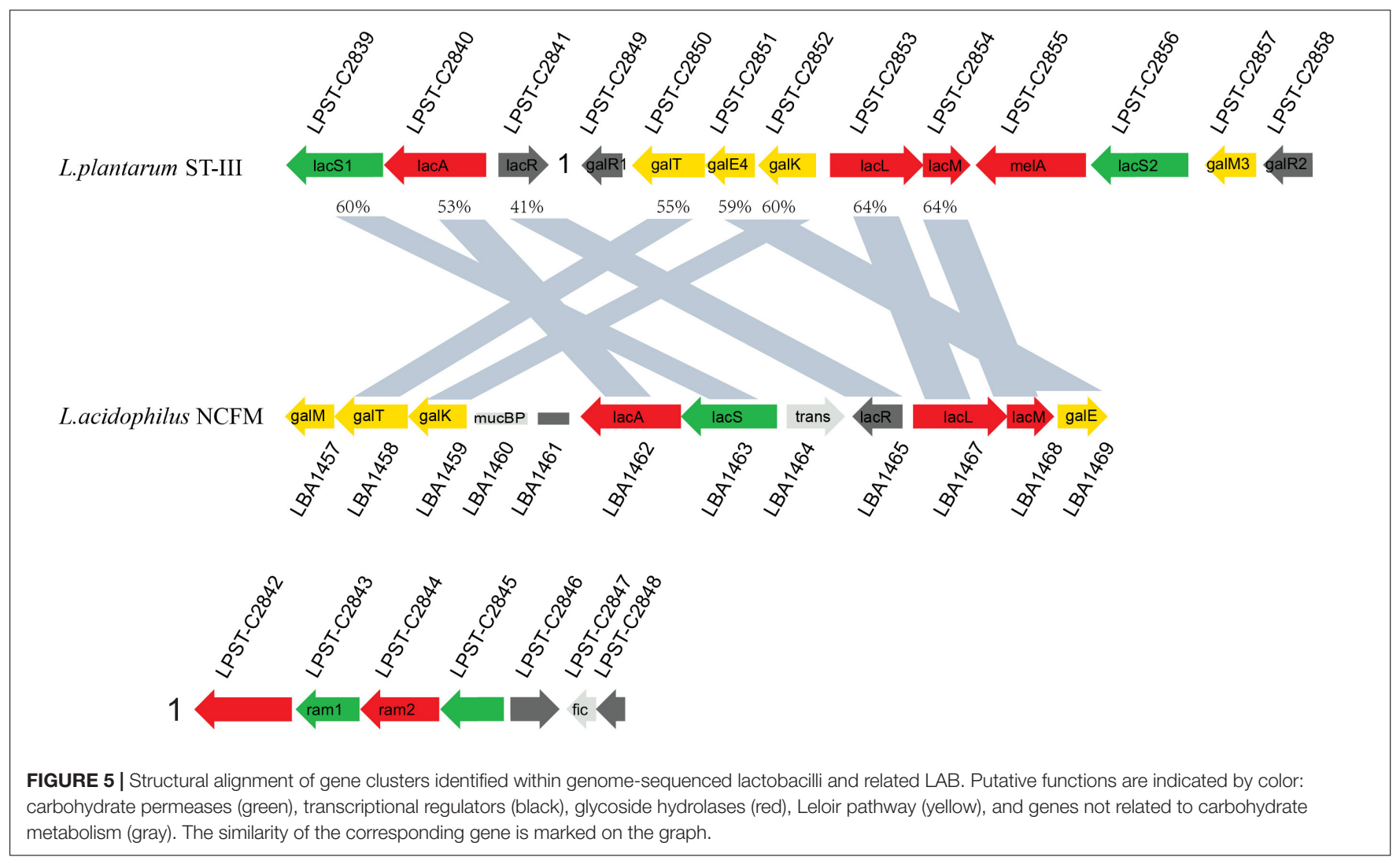




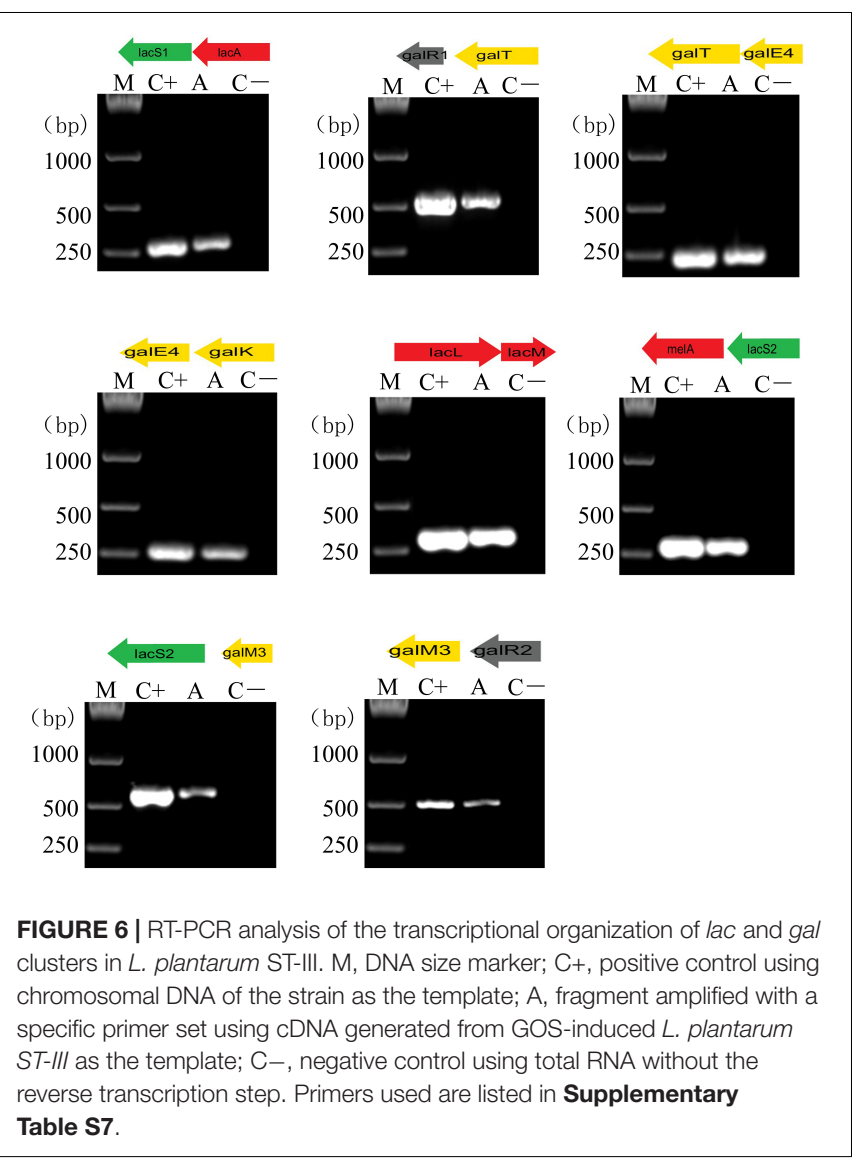

the PCR template (Chen et al., 2018). The results demonstrated that the gal cluster was transcribed into three polycistronic units: galR1, galT, galE4, and galK transcribed together; melA, lacS2, galM3, and galR2 cotranscribed as an operon; and lacL and lacM divergently oriented and transcribed together. As expected, the lac cluster was transcribed into two transcriptional units: lacS1 and lacA cotranscribed as an operon and lacR transcribed alone (Figure 6).

\section{Comparison Between the ccpA Mutant Grown on GOS and Glucose}

Next, the transcriptome of the $\operatorname{csp} A$ mutant in response to glucose and GOS was compared. Compared with the ccpA mutant grown on glucose, 223 genes (7\% of all genes) were found to be differentially expressed in the $c c p A$ mutant grown on GOS, 119 of which were upregulated and 104 were downregulated. Notably, 89 of the 223 genes could be clustered into special pathways (Figure 3A). Compared with the gene expression in the comparison between the wild-type strain grown on GOS and glucose, the significantly regulated genes in this comparison was significantly reduced, indicating that some differentially expressed genes were mainly regulated by CcpA. For instance, expression of almost none of the genes associated with pyruvate metabolism and fatty acid synthesis differed in this comparison as only functional CcpA can mediate their differential expression. Nonetheless, most of the differentially expressed genes (65\%) were also involved in carbohydrate metabolism (Figure 4). The differentially expressed genes related to carbohydrate metabolism, especially the PTS (only 10 genes), also significantly decreased under this comparison; these PTS were mainly involved in the transport of beta-glucose and mannitol (Supplementary Table S6). For glycolysis, most of the genes in this comparison showed the same changes relative to that of the wild-type grown on glucose and GOS.

As observed from the previous comparison, two gene clusters (lac and $g a l$ ) were upregulated in the wild-type strain grown on GOS compared with that grown on glucose. After CcpA knockout, GOS increased the expression of the lac gene cluster by at least 60.93-fold as well as that of the gal cluster, albeit to a lesser extent (at least 9.33-fold) (Supplementary Table S5). These results indicate that these genes may be controlled by regulators other than CcpA.

\section{Comparison Between the Wild-Type and ccpA Mutant Grown on GOS}

As reported previously, CcpA plays a key role in carbohydrate metabolism regulation in $L$. plantarum and functions in a glucose-dependent manner (Chen et al., 2018); thus, the difference in the transcriptome profile between wild-type and ccpA mutant was low (6\%) could be expected. Further, compared with the wild-type strain, approximately 187 genes (6\%) were differentially expressed in the $c c p A$ mutant at least by 2 -fold in the presence of GOS ( $p<0.05), 145$ of which were upregulated and 42 were downregulated (Figure 3B). Among these 187 genes, only 81 could be mapped to the metabolic pathways. Most of these genes were also mapped to carbohydrate metabolism (37\%), similar to those in the wild-type and $c c p A$ mutant grown on glucose (Chen et al., 2015). These genes are mainly involved in the PTS, pyruvate metabolism, glycolysis, and fructose and mannose metabolism (Figure 4).

Due to the loss of CcpA, GOS could also induce the expression of some genes encoding PTS components (2.35-20.20-fold increase), including sucrose-specific transporter, cellobiosespecific transporter, and galactitol-specific transporter. This manifested that these genes were also inhibited by GOS in the absence of CcpA. Notably, the expression of the lac and gal clusters did not differ significantly between the wild-type and cсpA mutant under the GOS condition. As both strains were grown on GOS, the genes involved in GOS metabolism were expressed similarly in both strains.

Similar to that observed in the comparison of wild-type and $\operatorname{ccp} A$ mutant under the glucose condition, some genes involved in the metabolism of pyruvate, including components of pyruvate dehydrogenase (LPST_C1776- LPST_C1778) and pyruvate oxidases (LPST_C2161), displayed similar trends in the presence of GOS. Interestingly, the gene (LPST_C0172) encoding acetate kinase was downregulated in the glucose condition but significantly upregulated in the GOS condition with CcpA inactivation. These results suggested that the presence of GOS could switch the fermentation pattern from homofermentation to heterofermentation, although to a lesser extent. The results of metabolite production confirm this hypothesis. 


\section{DISCUSSION}

The prebiotic effect of GOS on the enteric populations has been confirmed in vivo and in vitro (Burr et al., 2010; Andersen et al., 2011; Mazzeo et al., 2012; Akiyama et al., 2015; Safari and Paolucci, 2017). Moreover, the role of CcpA as a global regulator has been revealed in many carbon metabolism regulatory mechanisms in gram-positive bacteria (Zotta et al., 2012; Chen et al., 2018; Lu et al., 2018). However, our knowledge of the mechanism of GOS metabolism in L. plantarum, especially of the regulatory mechanism of CcpA, is still limited. In the present study, the whole transcriptome, growth profiles, and metabolite production of L. plantarum ST-III and L. plantarum $\triangle c c p A$ were analyzed using GOS or glucose as the sole carbohydrate source to reveal the global changes in response to carbon sources and availability.

The presence of GOS significantly affected the growth of L. plantarum ST-III, consistent with the findings of its growth on FOS in our previous study (Chen et al., 2015). The $\mu_{\max }$ results indicated that the wild-type strain grown on oligosaccharides, including FOS and GOS, showed significantly reduced growth compared with that grown on glucose. After $c c p A$ deletion, $\mu_{\max }$ further declined in the $\operatorname{ccp} A$ mutant compared with that in the wild-type strain grown on the same carbon sources. Notably, the absence of $\operatorname{ccp} A$ (mutant strain) leaded to a slow downward growth trend in the stationary phase in both carbon sources, whereas the presence of $\operatorname{ccp} A$ (wild-type strain) slowly increased the growth. These results of growth experiment indicate that the growth of L. plantarum differed between GOS and glucose and that CcpA was a key contributor to this difference. Whole transcriptome sequencing better revealed the differential gene expression of $L$. plantarum ST-III grown on different carbon sources. Among the four pair-wise comparisons, the maximum number of differentially expressed genes was observed in the comparison of the wild-type strain grown on glucose and GOS, whereas the comparison between the $\operatorname{ccpA}$ mutant grown on glucose and GOS showed fewer differentially expressed genes. These results confirm that CcpA plays a key role in carbohydrate metabolism regulation in L. plantarum. Furthermore, the lowest number of differentially expressed genes was observed in the comparison of wild-type and $c с p A$ mutant under the GOS condition. Studies have shown that carbohydrate metabolism regulation in L. plantarum is mainly mediated in a glucose-dependent manner (Gorke and Stulke, 2008; Cai et al., 2012). Although GOS is considered to be a poorly metabolized carbohydrate, it can also trigger the regulation of carbon catabolic metabolites (Zeng et al., 2013).

As mentioned above, almost all genes of the lac and gal clusters were significantly upregulated (2.93-284.05-fold) in L. plantarum ST-III using GOS or glucose as the sole carbohydrate source. This finding indicates that these two clusters may be involved in GOS metabolism. Furthermore, BLAST analysis revealed that these clusters are similar to those of $L$. acidophilus NCFM, with the exception that L. plantarum has two more copy of the melA gene and lacS2 gene. RT-PCR analysis of the transcriptional organization of these gene clusters indicated that the gene cluster associated with GOS metabolism comprises five independent polycistronic units. Previous studies have shown that in the presence of a preferred carbon source, usually glucose, CcpA can effectively bind to cre sites to inhibit or activate the transcription of non-preferred metabolism genes (Huichun et al., 2012; Wu et al., 2015). In this study, four cre sites were identified among the two clusters (Figure 7), suggesting that the upregulation of these clusters is attributable to the binding of CcpA to cre sites within or downstream of the promoter. Notably, the two clusters were also upregulated (9.33-60.93-fold) when comparing ccpA mutant cells grown on GOS and glucose, indicating that except for CcpA, GOS metabolism may also be affected by other local regulators.

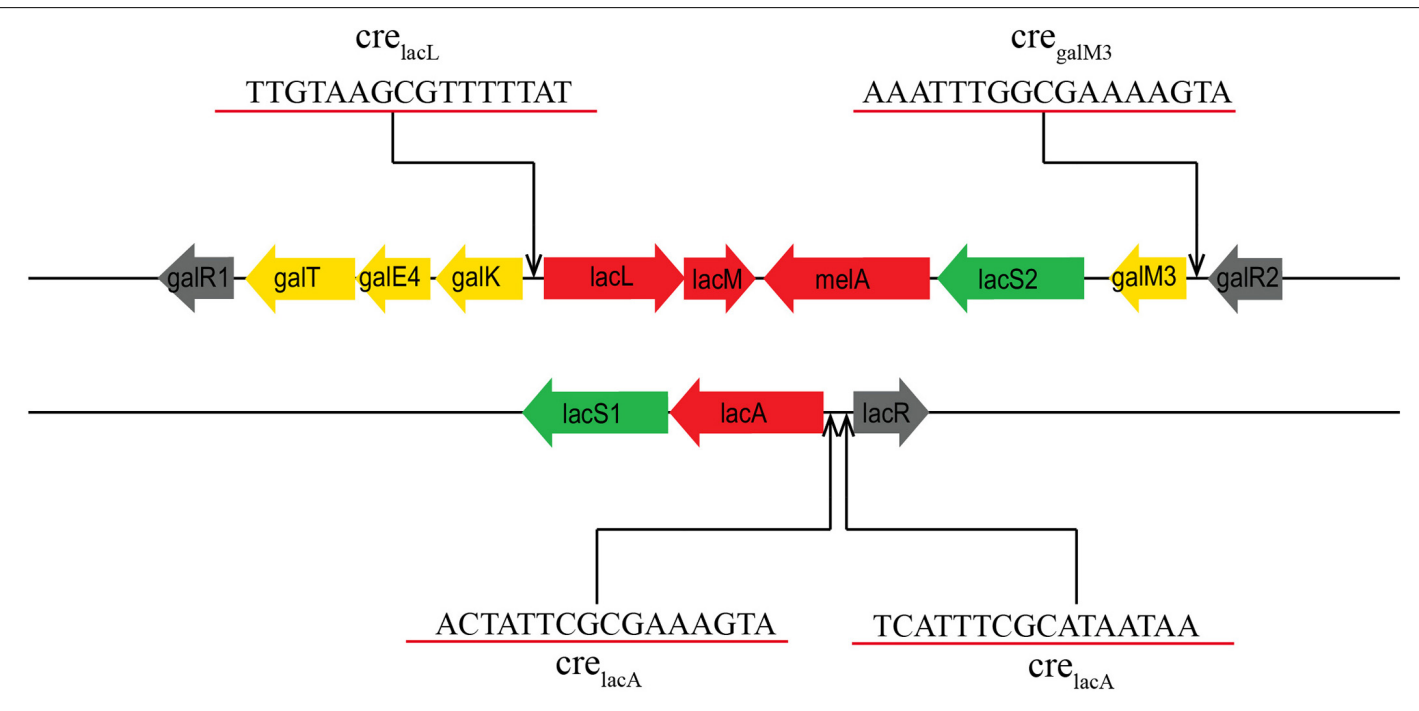

FIGURE 7 | Prediction of the potential cre sites in the gal and lac clusters of L. plantarum ST-III. Putative functions are indicated by color: carbohydrate permease (green), transcriptional regulators (black), glycoside hydrolases (red), Leloir pathway (yellow). Putative cre sites are underlined in red. 
Furthermore, three genes encoding the regulatory factors of LacI family, including lacR1 (LPST-C2841), galR1 (LPST-C2849), and galR2 (LPST-C2858), were found in the gene clusters associated with GOS metabolism. A detailed investigation of the GOS regulatory mechanism in L. plantarum is currently underway.

One interesting outcome of this study concerns pyruvate metabolism. Genes related to the proposed pathways for the production of acetate and formate were upregulated in the presence of GOS versus glucose. The measurement of metabolites showed that cells grown on GOS altered their metabolism to produce more acetate and less lactate. These results were similar to our previous study with L. plantarum grown on FOS (Chen et al., 2015). Furthermore, after CcpA knockout, the cсpA mutant grown on glucose or GOS produced more acetate and less lactate than the wild-type strain. These results confirm that the inactivation of $c c p A$ leads to a shift from homolactic fermentation to mixed fermentation. It is suggested that the shift from homolactic fermentation to mixed fermentation by L. plantarum is affected by many factors, e.g., ccpA inactivation, sugar exhaustion and aerobic condition (Lorquet et al., 2004; Mazzeo et al., 2012; Zotta et al., 2012). As these factors all existed in the present work, thus it can be concluded that the metabolic shifts observed could be attributed to the interaction of these factors.

The transcriptome data suggested that there are several common characteristics between FOS and GOS metabolisms in L. plantarum. For example, the gene clusters involved in oligosaccharide metabolism were significantly upregulated in the presence of FOS or GOS, suggesting that these genes are induced for oligosaccharide utilization. In addition, the transcriptome profiles indicated that the fatty acid synthesis-related genes were significantly downregulated in the wild-type cells grown on GOS compared with those on glucose. The result is consistent with the finding of our previous study that oligosaccharide metabolism decreases FA synthesis (Chen et al., 2015). Interestingly, this phenomenon was eliminated after the $c c p A$ gene was knocked out, which suggested that CcpA also has an important effect on the regulation of fatty acid metabolism (Lu et al., 2018). Furthermore, the differentially expressed genes of pyruvate metabolism and measurement of metabolites in L. plantarum grown on GOS were also similar to our previous studies using FOS as the carbon source (Chen et al., 2015; Lu et al., 2018). In summary, many similar phenomena and changes were observed in the metabolism of different oligosaccharides in L. plantarum, including differential expression of related metabolic genes, conversion of organic acids, and changes in fatty acid metabolism, and CcpA was found to play an important role in these processes. Thus, our results suggest that L. plantarum exhibits a similar adaptation mechanism in response to oligosaccharides, but the detailed mechanism needs to be investigated in future studies.

\section{REFERENCES}

Akiyama, T., Kimura, K., and Hatano, H. (2015). Diverse galactooligosaccharides consumption by bifidobacteria: implications of $\beta$-galactosidase-LacS operon. Biosci. Biotechnol. Biochem. 79, 664-672. doi: 10.1080/09168451.2014.987204

\section{CONCLUSION}

In conclusion, we performed transcriptome and metabolite analyses of $L$. plantarum and L. plantarum $\triangle c c p A$ grown on glucose and GOS. The results demonstrated that the lac and gal gene clusters resemble the corresponding clusters in $L$. acidophilus NCFM that are involved in GOS utilization and that the same clusters may participate in GOS metabolism in L. plantarum. In addition, these clusters contain five independent polycistronic units and four potential cre sites for CcpA binding. Meanwhile, we found many common characteristics between FOS and GOS metabolisms in L. plantarum, including the induction of oligosaccharide-related genes, conversion to mixed fermentation, and changes in fatty acid biosynthesis. The data obtained in this in vitro study provide insight about the mechanism of GOS metabolism in $\mathrm{LAB}$ and contribute to the elucidation of complex regulatory networks that function in response to different carbon sources and availability.

\section{DATA AVAILABILITY}

Publicly available datasets were analyzed in this study. This data can be found here: https://www.ncbi.nlm.nih.gov/search/all/ ?term=+PRJNA493968.

\section{AUTHOR CONTRIBUTIONS}

CC wrote the manuscript and performed the statistical analyses. LW analyzed the growth and transcriptome of wild-type and $\operatorname{csp} A$ mutant in response to glucose and GOS. YL constructed the $\operatorname{ccp} A$ mutant and analyzed the generalizability of oligosaccharide metabolism. HY performed the RT-PCR analysis, BLASTp analysis, and putative cre site prediction. HT designed the research.

\section{FUNDING}

This work was supported by the National Natural Science Foundation of China (Award 31501451).

\section{SUPPLEMENTARY MATERIAL}

The Supplementary Material for this article can be found online at: https://www.frontiersin.org/articles/10.3389/fmicb. 2019.01584/full\#supplementary-material

Andersen, J. M., Barrangou, R., Hachem, M. A., Lahtinen, S., Yong, J. G., Svensson, B., et al. (2011). Transcriptional and functional analysis of galactooligosaccharide uptake by lacS in Lactobacillus acidophilus. Proc. Natl. Acad. Sci. U.S.A. 108, 17785-17790. doi: 10.1073/pnas.1114 152108 
Andersen, J. M., Barrangou, R., Hachem, M. A., Lahtinen, S. J., Goh, Y. J., Svensson, B., et al. (2012). Transcriptional analysis of prebiotic uptake and catabolism by Lactobacillus acidophilus NCFM. PLoS One 7:e44409. doi: 10.1371/journal. pone.0044409

Arena, M. P., Capozzi, V., Longo, A., Russo, P., Rieu, A., Guzzo, J., et al. (2019). The phenotypic analysis of Lactobacillus plantarum shsp mutants reveals a potential role for hsp1 in cryotolerance. Front. Microbiol. 10:838. doi: 10.3389/fmicb. 2019.00838

Arnold, J., Simpson, J., Roach, J., Bruno-Barcena, J., and Azcarate-Peril, M. (2018). Prebiotics for lactose intolerance: variability in galacto-oligosaccharide utilization by intestinal Lactobacillus rhamnosus. Nutrients 10:1517. doi: 10.3390/nu10101517

Azcarate-Peril, M. A., Ritter, A. J., Savaiano, D., Monteagudo-Mera, A., Anderson, C., Magness, S. T., et al. (2017). Impact of short-chain galactooligosaccharides on the gut microbiome of lactose-intolerant individuals. Proc. Natl. Acad. Sci. U.S.A. 114, E367-E375. doi: 10.1073/pnas.1606722113

Burr, G., Hume, M., Ricke, S., Nisbet, D., and Gatlin, D. (2010). In vitro and in vivo evaluation of the prebiotics GroBiotic ${ }^{\circledR}-\mathrm{A}$, inulin, mannanoligosaccharide, and galactooligosaccharide on the digestive microbiota and performance of hybrid striped bass (Morone chrysops $\times$ Morone saxatilis). Microb. Ecol. 59, 187-198. doi: 10.1007/s00248-009-9597-6

Cai, J., Tong, H., Qi, F., and Dong, X. (2012). CcpA-dependent carbohydrate catabolite repression regulates galactose metabolism in Streptococcus oligofermentans. J. Bacteriol. 194, 3824-3832. doi: 10.1128/JB.00156-12

Capozzi, V., Russo, P., Ladero, V., Fernández, M., Fiocco, D., Alvarez, M. A., et al. (2012). Biogenic amines degradation by Lactobacillus plantarum: toward a potential application in wine. Front. Microbiol. 3:122. doi: 10.3389/fmicb.2012. 00122

Carvalho, S. M., Kloosterman, T. G., Kuipers, O. P., and Neves, A. R. (2011). CcpA ensures optimal metabolic fitness of Streptococcus pneumoniae. PLoS One 6:e026707. doi: 10.1371/journal.pone.0026707

Chen, C., Guozhong, Z., Wei, C., and Benheng, G. (2015). Metabolism of fructooligosaccharides in Lactobacillus plantarum ST-III via differential gene transcription and alteration of cell membrane fluidity. Appl. Environ. Microbiol. 81, 7697-7707. doi: 10.1128/AEM.02426-15

Chen, C., Lu, Y., Wang, L., Yu, H., and Tian, H. (2018). CcpA-dependent carbon catabolite repression regulates fructooligosaccharides metabolism in Lactobacillus plantarum. Front. Microbiol. 9:1114. doi: 10.3389/fmicb.2018. 01114

Enan, G., El-Essawy, A. A., Uyttendaele, M., and Debevere, J. (1996). Antibacterial activity of Lactobacillus plantarum UG1 isolated from dry sausage: characterization, production and bactericidal action of plantaricin UG1. Int. J. Food Microbiol. 30, 189-215. doi: 10.1016/0168-1605(96)00947-6

Fujita, Y. (2009). Carbon catabolite control of the metabolic network in Bacillus subtilis. J. Agric. Chem. Soc. Jpn. 73, 245-259. doi: 10.1271/bbb.80479

Goffin, P., Muscariello, L., Lorquet, F., Stukkens, A., Prozzi, D., Sacco, M., et al. (2006). Involvement of pyruvate oxidase activity and acetate production in the survival of Lactobacillus plantarum during the stationary phase of aerobic growth. Appl. Environ. Microbiol. 72, 7933-7940. doi: 10.1128/AEM.00659-06

Goh, Y. J., and Klaenhammer, T. R. (2015). Genetic mechanisms of prebiotic oligosaccharide metabolism in probiotic microbes. Annu. Rev. Food Sci. Technol. 6, 137-156. doi: 10.1146/annurev-food-022814-015706

Gorke, B., and Stulke, J. (2008). Carbon catabolite repression in bacteria: many ways to make the most out of nutrients. Nat. Rev. Microbiol. 6, 613-624. doi: $10.1038 /$ nrmicro1932

Huichun, T., Fengxia, Q., and Xiuzhu, D. (2012). CcpA-dependent carbohydrate catabolite repression regulates galactose metabolism in Streptococcus oligofermentans. J. Bacteriol. 194, 3824-3832. doi: 10.1128/JB.00156-12

Iqbal, S., Nguyen, T. H., Nguyen, T. T., Maischberger, T., and Haltrich, D. (2010). $\beta$-Galactosidase from Lactobacillus plantarum WCFS1: biochemical characterization and formation of prebiotic galacto-oligosaccharides. Carbohydr. Res. 345, 1408-1416. doi: 10.1016/j.carres.2010.03.028

Josef, D., Christof, F., and Postma, P. W. (2006). How phosphotransferase systemrelated protein phosphorylation regulates carbohydrate metabolism in bacteria. Microbiol. Mol. Biol. Rev. Mmbr. 70, 939-1031. doi: 10.1128/MMBR.00024-06

Kremling, A., Geiselmann, J., Ropers, D., and De, J. H. (2015). Understanding carbon catabolite repression in Escherichia coli using quantitative models. Trends Microbiol. 23, 99-109. doi: 10.1016/j.tim.2014.11.002
Langmead, B., Trapnell, C., Pop, M., and Salzberg, S. L. (2009). Ultrafast and memory-efficient alignment of short DNA sequences to the human genome. Genome Biol. 10:R25. doi: 10.1186/gb-2009-10-3-r25

Lorquet, F., Goffin, P., Muscariello, L., Baudry, J., Ladero, V., Sacco, M., et al. (2004). Characterization and functional analysis of the poxB Gene, which encodes pyruvate oxidase in Lactobacillus plantarum. J. Bacteriol. 186, 37493759. doi: $10.1074 /$ jbc.M400439200

Lu, Y., Song, S., Tian, H., Yu, H., Zhao, J., and Chen, C. (2018). Functional analysis of the role of CcpA in Lactobacillus plantarum grown on fructooligosaccharides or glucose: a transcriptomic perspective. Microb. Cell Fact. 17:201. doi: 10.1186/ s12934-018-1050-4

Macfarlane, G. T., Steed, H., and Macfarlane, S. (2008). Bacterial metabolism and health-related effects of galacto-oligosaccharides and other prebiotics. J. Appl. Microbiol. 104, 305-344. doi: 10.1111/j.1365-2672.2007.03520.x

María, I. P., Analía, I. E., and Manrique, G. D. (2018). Fermentation by Lactobacillus paracasei of galactooligosaccharides and low-molecular-weight carbohydrates extracted from squash (Curcubita maxima) and lupin (Lupinus albus) seeds. J. Microbiol. Biotechnol. Food Sci. 3:329.

Mazzeo, M. F., Cacace, G., Peluso, A., Zotta, T., Muscariello, L., Vastano, V., et al. (2012). Effect of inactivation of ccpA and aerobic growth in Lactobacillus plantarum: a proteomic perspective. J. Proteo. 75, 4050-4061. doi: 10.1016/j. jprot.2012.05.019

Monteagudo-Mera, A., Arthur, J. C., Jobin, C., Keku, T., Bruno-Barcena, J. M., and Azcarate-Peril, M. A. (2016). High purity galacto-oligosaccharides enhance specific Bifidobacterium species and their metabolic activity in the mouse gut microbiome. Benef. Microbes 7, 247-264. doi: 10.3920/BM2015.0114

Muscariello, L., Marasco, R., De Felice, M., and Sacco, M. (2001). The functional ccpA gene is required for carbon catabolite repression in Lactobacillus plantarum. Appl. Environ. Microbiol. 67, 2903-2907. doi: 10.1128/AEM.67.7. 2903-2907.2001

Muscariello, L., Vastano, V., Siciliano, R. A., Sacco, M., and Marasco, R. (2011). Expression of the Lactobacillus plantarum malegene is regulated by CcpA and a malr-like protein. J. Microbiol. 49, 950-955. doi: 10.1007/s12275-011-0495-5

Papadopoulou, O. S., Argyri, A. A., Varzakis, E. E., Tassou, C. C., and Chorianopoulos, N. G. (2018). Greek functional feta cheese: enhancing quality and safety using a Lactobacillus plantarum strain with probiotic potential. Food Microbiol. 74, 21-33. doi: 10.1016/j.fm.2018.02.005

Pavli, F. G., Argyri, A. A., Papadopoulou, O. S., Nychas, G. J. E., Chorianopoulos, N. G., and Tassou, C. C. (2016). Probiotic potential of lactic acid bacteria from traditional fermented dairy and meat products: assessment by in vitro tests and molecular characterization. J. Prob. Health 4:157. doi: 10.4172/2329-8901. 1000157

Presecan-Siedel, E., Galinier, A., Longin, R., Deutscher, J., Danchin, A., Glaser, P., et al. (1999). Catabolite regulation of the pta gene as part of carbon flow pathways in Bacillus subtilis. J. Bacteriol. 181:6889. doi: 10.1076/noph.25.3.163. 7128

Qian, L., Xueqin, N., Qiang, W., Zhirong, P., Lili, N., Hengsong, W., et al. (2017). Lactobacillus plantarum bsgp201683 isolated from giant panda feces attenuated inflammation and improved gut microflora in mice challenged with enterotoxigenic Escherichia coli. Front. Microbiol. 8:1885. doi: 10.3389/fmicb. 2017.01885

Ren, Q., Xu, Z. L., Wang, X. W., Zhao, X. F., and Wang, J. X. (2009). Clip domain serine protease and its homolog respond to vibrio challenge in Chinese white shrimp, Fenneropenaeus chinensis. Fish Shellish Immunol. 26, 787-798. doi: 10.1016/j.fsi.2009.03.004

Ricciardi, A., Guidone, A., Ianniello, R. G., Cioffi, S., Aponte, M., Pavlidis, D., et al. (2015). A survey of non-starter lactic acid bacteria in traditional cheeses: culture dependent identification and survival to simulated gastrointestinal transit. Int. Dairy J. 43, 42-50. doi: 10.1016/j.idairyj.2014. 11.006

Robert, H., Marrec, C. L., Blanco, C., and Jebbar, M. (2000). Glycine betaine, carnitine, and choline enhance salinity tolerance and prevent the accumulation of sodium to a level inhibiting growth of Tetragenococcus halophila. Appl. Environ. Microbiol. 66, 509-517. doi: 10.1128/AEM.66.2.509-517.2000

Safari, O., and Paolucci, M. (2017). Effect of in vitro selected synbiotics (galactooligosaccharide and mannanoligosaccharide with or without Enterococcus faecalis) on growth performance, immune responses and intestinal microbiota of juvenile narrow clawed crayfish, Astacus 
leptodactylus leptodactylus Eschscholtz, 1823. Aquacult. Nutr. 24, 247-259. doi: 10.1111/anu.12553

Sankar, G. S., Sankar, S. S., Subrata, S., Venkatachalam, S., and Chang, P. S. (2018). Use of a potential probiotic, Lactobacillus plantarum L7, for the preparation of a rice-based fermented beverage. Front. Microbiol. 9:473. doi: 10.3389/fmicb. 2018.00473

Seidl, K., Müller, S., François, P., Kriebitzsch, C., Schrenzel, J., Engelmann, S., et al. (2009). Effect of a glucose impulse on the CcpA regulon in Staphylococcus aureus. BMC Microbiol. 9:95. doi: 10.1186/1471-2180-9-95

Siezen, R., Boekhorst, J., Muscariello, L., Molenaar, D., Renckens, B., and Kleerebezem, M. (2006). Lactobacillus plantarumgene clusters encoding putative cell-surface protein complexes for carbohydrate utilization are conserved in specific gram-positive bacteria. BMC Genomics 7:126. doi: 10.1186/1471-2164-7-126

Tan, Y., Liu, Z. Y., Liu, Z., Zheng, H. J., and Li, F. L. (2015). Comparative transcriptome analysis between csrA-disruption Clostridium acetobutylicum and its parent strain. Mol. Biosyst. 11, 1434-1442. doi: 10.1039/c4mb00600c

Teusink, B., Enckevort, F. H. J. V., Francke, C., Wiersma, A., Wegkamp, A., Smid, E. J., et al. (2005). In silico reconstruction of the metabolic pathways of Lactobacillus plantarum: comparing predictions of nutrient requirements with those from growth experiments. Appl. Environ. Microbiol. 71, 7253-7262. doi: 10.1128/AEM.71.11.7253-7262.2005

Titgemeyer, F., and Hillen, W. (2002). Global control of sugar metabolism: a gram-positive solution. Antonie Van Leeuwenhoek 82, 59-71. doi: 10.1023/A: 1020628909429

Van Leeuwen, S. S., Kuipers, B. J. H., Dijkhuizen, L., and Kamerling, J. P. (2016). Comparative structural characterization of 7 commercial galactooligosaccharide (gos) products. Carbohydr. Res. 425, 48-58. doi: 10.1016/j. carres.2016.03.006

Wang, L., Feng, Z., Wang, X., Wang, X., and Zhang, X. (2010). DEGseq: an $\mathrm{R}$ package for identifying differentially expressed genes from RNA-seq data. Bioinformatics 26, 136-138. doi: 10.1093/bioinformatics/btp612

Wang, T., Teng, K., Liu, Y., Shi, W., Zhang, J., Zhang, X., et al. (2019). Lactobacillus plantarum PFM 105 promotes intestinal development through modulation of gut microbiota in weaning piglets. Front. Microbiol. 10:90. doi: 10.3389/fmicb. 2017.01885

Wang, Y., Chen, C., Ai, L., Zhou, F., Zhou, Z., Wang, L., et al. (2011). Complete genome sequence of the probiotic Lactobacillus plantarum ST-III. J. Bacteriol. 193, 313-314. doi: 10.1128/JB.01159-10
Wu, Y., Yang, Y., Ren, C., Yang, C., Yang, S., Gu, Y., et al. (2015). Molecular modulation of pleiotropic regulator CcpA for glucose and xylose coutilization by solvent-producing Clostridium acetobutylicum. Metab. Eng. 28, 169-179. doi: 10.1016/j.ymben.2015.01.006

Yadav, R., Puniya, A. K., and Shukla, P. (2016). Probiotic properties of Lactobacillus plantarum RYPR1 from an indigenous fermented beverage raabadi. Front. Microbiol. 7:1683. doi: 10.3389/fmicb.2016.01683

Yong, J. G., and Klaenhammer, T. R. (2015). Genetic mechanisms of prebiotic oligosaccharide metabolism in probiotic microbes. Annu. Rev. Food Sci. Technol. 6, 137-156. doi: 10.1146/annurev-food-022814-015706

Zeng, L., Choi, S. C., Danko, C. G., Siepel, A., Stanhope, M. J., and Burne, R. A. (2013). Gene regulation by CcpA and catabolite repression explored by RNASeq in Streptococcus mutans. PLoS One 8:e060465. doi: 10.1371/journal.pone. 0060465

Zhang, Z. Y., Liu, C., Zhu, Y. Z., Zhong, Y., Zhu, Y. Q., Zheng, H. J., et al. (2009). Complete Genome Sequence of Lactobacillus plantarum JDM1. J. Bacteriol. 191, 5020-5021. doi: 10.1128/JB.00587-09

Zhao, H., Liu, L., Peng, S., Yuan, L., Li, H., and Wang, H. (2019). Heterologous expression of argininosuccinate synthase from Oenococcus oeni enhances the acid resistance of Lactobacillus plantarum. Front. Microbiol. 10:1393. doi: $10.3389 /$ fmicb.2019.01393

Zheng, H., Liu, E., Shi, T., Ye, L., Konno, T., Oda, M., et al. (2015). Strandspecific RNA-seq analysis of the Lactobacillus delbrueckii subsp. bulgaricus transcriptome. Mol. Biosyst. 12:508. doi: 10.1039/C5MB00547G

Zotta, T., Ricciardi, A., Guidone, A., Sacco, M., Muscariello, L., Mazzeo, M. F., et al. (2012). Inactivation of and aeration affect growth, metabolite production and stress tolerance in Lactobacillus plantarum WCFS1. Int. J. Food Microbiol. 155, 51-59. doi: 10.1016/j.ijfoodmicro.2012.01.017

Conflict of Interest Statement: The authors declare that the research was conducted in the absence of any commercial or financial relationships that could be construed as a potential conflict of interest.

Copyright (c) 2019 Chen, Wang, Lu, Yu and Tian. This is an open-access article distributed under the terms of the Creative Commons Attribution License (CC BY). The use, distribution or reproduction in other forums is permitted, provided the original author(s) and the copyright owner(s) are credited and that the original publication in this journal is cited, in accordance with accepted academic practice. No use, distribution or reproduction is permitted which does not comply with these terms. 